\title{
Highlights of the European Society of Musculoskeletal Radiology (ESSR) annual meeting 2009
}

\author{
Andrew J. Grainger
}

Published online: 14 November 2009

(C) ISS 2009

\section{Introduction}

The 16th annual congress of the European Society of Musculoskeletal Radiology (ESSR) took place in Genoa, Italy, on 12 and 13 June 2009. The meeting was held jointly with the Italian Congress of Musculoskeletal Radiology, which took place on 11 June. A successful musculoskeletal ultrasound course was also run in the afternoon of 11 June, organised by the society's ultrasound subcommittee.

As usual, the meeting comprised a refresher course, which, this year, took the hip and bony pelvis as its theme, scientific sessions, special-focus sessions and a scientific e-poster exhibition. There were 688 delegates attending the ESSR meeting, coming from right across Europe and beyond, while 703 attended the Italian Society meeting. In total 181 electronic posters were exhibited, and 49 scientific presentations were made. The posters and presentations covered the whole spectrum of musculoskeletal imaging, including novel technologies and techniques and new perspectives on wellrecognised conditions. The abstracts of papers presented at the meeting appeared in the June 2009 edition of Skeletal Radiology [1]. This article attempts to highlight some of the presentations that might be of interest.

\section{Techniques and technology}

As might be expected, a number of papers focused on applications for $3 \mathrm{~T}$ magnetic resonance (MR) imaging. A

\section{A. J. Grainger $(\triangle)$}

Musculoskeletal Centre,

Chapeltown Road,

Leeds LS7 4SA, UK

e-mail: andrew.grainger@leedsth.nhs.uk group from Munich published their first results of the use of an isotropic three-dimensional (3D) turbo-spin echo (TSE) sequence for imaging the knee [2]. Total imaging time for the sequence was in the order of $12.5 \mathrm{~min}$, and the readers used $1 \mathrm{~mm}$-thick multiplanar reconstructions for review. They found the 3D sequence images to be at least comparative with state-of-the-art two-dimensional (2D) sequences, but, of course, they had the advantage of being able to undertake oblique reconstructions or review of thinner slices. A group from Ireland and the Netherlands showed that $3 \mathrm{~T}$ magnetic resonance imaging (MRI) was able to identify the double-bundle anatomy of the anterior cruciate ligament (ACL) in $94 \%$ of patients in a retrospective review of 50 patients with intact ACLs [3]; while the utility of 3 T MRI for evaluating upper limb nerve structure using diffusion imaging and tractography was demonstrated by an Austrian group [4].

In an interesting study looking at the effects of cartilage loading and unloading on T1-weighted gadolinium-enhanced (T1-Gd) relaxation times using the gadolinium-enhanced magnetic resonance imaging of cartilage (dGEMRIC) technique, workers showed that loading articular cartilage reduced the mean T1-Gd relaxation time by approximately $50 \mathrm{~ms}$ [5]. They emphasised that this needed to be considered when the dGEMRIC technique is to be used to assess glycosaminoglycan depletion in cartilage disease, which is also seen as a reduction in $\mathrm{T} 1-\mathrm{Gd}$ relaxation time.

A study from Switzerland looked at the use of advanced MRI techniques for the diagnosis and prediction of outcome in patients with Perthes' disease [6]. They used diffusion-weighted MRI and dynamic gadolinium-enhanced subtraction techniques, comparing them with pinhole scintigraphy. They identified increased apparent diffusion coefficients (ADCs) in the femoral epiphyses as being sensitive and specific for the diagnosis, but they found that 
increased $\mathrm{ADC}$ in the femoral metastasis predicted a poor clinical outcome.

\section{Bone and soft tissue tumours}

This year's prize for the best presentation at the meeting went to a group from Birmingham in the UK who presented two related papers highlighting the imaging appearances following hindquarter amputation both in the normal post-operative situation and in cases of local disease recurrence $[7,8]$. Given that this is a procedure reserved for patients with large bone or soft tissue sarcomas that cannot be treated by compartment resection or less radical amputation, the group had amassed a relatively large series in their retrospective review. Eighteen patients had been identified who had suffered no recurrent tumours or complications following the surgery. In all surviving patients the intensity of the MRI signal in the muscle flap had normalised within 4 years, although, initially, all subjects had shown high signal intensities on T2-weighted/short-tau inversion recovery (T2/STIR) sequences here. Of the patients, $20 \%$ had shown fat infiltration into the muscle flap within the first 6 months of the operation, and, within 2 years, all patient showed this feature. Fat atrophy was seen in all patients studied after 1 year. In 20 patients where follow-up examination demonstrated recurrent disease or pelvic metastases, the majority of recurrences $(43 \%)$ occurred in the muscle component of the amputation flap. In all cases the signal intensity pattern of the recurrent tumour mirrored that of the original tumour.

MRI is not readily available across all of Europe, and it is always interesting to see approaches that use different imaging technologies to solve common problems. A small study from the Czech Republic showed that computed tomography (CT) perfusion studies can be used to assess the sensitivity of osteosarcoma to preadjuvant chemotherapy with a high level of agreement with the histological findings [9].

\section{Imaging of arthritis}

The ESSR has an active arthritis subcommittee and always attracts papers from workers in the field of arthritis. A retrospective review of clinical data, laboratory tests and contrast-enhanced MRI of the sacroiliac joints in nearly 180 patients was undertaken by groups from Austria and Sweden to identify features on sacroiliac joint MRI that were more specific to ankylosing spondylitis than other causes of sacroiliitis or mechanical sacroiliac disease [10]. They found that irregularities of joint space, bone marrow oedema, subcortical cysts and contrast enhancement were inconsistently present in all patients. However, the presence of erosions was a more specific feature of ankylosing spondylitis.

Increasingly, studies are looking at the use of MRI in osteoarthritis, and two papers were presented from the Netherlands that described the utilisation of MRI in the assessment of knee osteoarthritis (OA). In one prospective study the group studied the significance of lesions of the anterior cruciate ligament and the meniscus as risk factors for knee osteoarthritis, after a mean follow-up period of 10 years [11]. The data showed that both meniscal and ACL tears were significant risk factors for the development of OA. A second study looked at the association of pain and disability in early knee osteoarthritis with joint abnormalities shown by MRI [12]. The study was composed of 155 patients with clinical evidence of early knee $\mathrm{OA}$ and 30 age and gender-matched controls. Features of OA on MRI were more common in the patient group, although they were seen in the control population, and no specific feature could distinguish between the two populations. Osteophyte and meniscal subluxation were MRI features most associated with symptoms in the patient population.

It is recognised that aromatase inhibitors used in the treatment of breast cancer are associated with an arthralgia syndrome. A team from Belgium looked at the MRI appearances of the hand and wrist joints in a group of patients at baseline and at 6 months after commencement of aromatase inhibitor therapy [13]. They found that the radiological findings were subtle, but they identified tendon tenosynovitis, soft tissue oedema and joint synovitis as features of the syndrome.

\section{Interventional techniques}

Several papers were presented in the field of interventional musculoskeletal radiology. Many of these related to ultrasound-guided procedures. Two studies focused on the treatment of calcific tendon disease. An Italian group described a large series of patients who had undergone ultrasound-guided needle aspiration/lavage of rotator cuff calcific tendinopathy [14]. They found that a large number of patients needed a second procedure to achieve benefit. A paper from Canada and Austria presented the results of calcium aspiration/lavage using a two-needle technique in a variety of tendons, the majority being rotator cuff tendons [15]. They used radiographs to assess the reduction in calcium following the procedure at a mean follow-up period of 12 weeks. They found a good clinical outcome in $69 \%$ of patients, all of whom showed a reduction in calcium area on the radiographs. The studies indicated that, when there was a 
resolution of greater than $60 \%$ of the calcification seen on radiographs, clinical improvement was seen. A UK study demonstrated the efficacy of ultrasound-guided therapy for plantar fasciitis, with $78 \%$ of patients studied showing a sustained benefit 12 months after the procedure [16]. No difference was found between patients receiving steroid alone and those undergoing dry needling prior to steroid injection. A different UK group showed the positive impact on patient treatment achieved by aspirating hip effusions in a paediatric population [17]. Not only did the aspiration allow the exclusion of infection, but, even in patients with no infection (transient synovitis), aspiration produced a relief in symptoms and a reduction in hospital stay.

\section{References}

1. 16th Annual Congress of the European Society of Musculoskeletal Radiology (ESSR). Genoa, Italy-June 11-13, 2009. Skeletal Radiol. 2009;38:603-31.

2. Notohamiprodjo N, Horng A, Pietschmann M, Horger W, Raya J, Park J, et al. MRI of the knee at $3 \mathrm{~T}$ : first clinical results with an isotropic PDfs-weighted 3-D-TFE-sequence [Abstract]. Skeletal Radiol. 2009;38:624.

3. Adriaensen MEAPM, Hogan BA, Al Bulushi HIJ, Kavanagh EC. Double bundle ACL anatomy at 3 tesla [Abstract]. Skeletal Radiol. 2009;38:603.

4. Kasprian G, Kainberger F, Brugger P, Engel H, Stuhr F, Aßmann $\mathrm{O}$, et al. Advanced $3 \mathrm{~T}$ MRI of upper extremity nerves [Abstract]. Skeletal Radiol. 2009;38:627-8.

5. Mayerhoefer ME, Weosch GH, Mamisch TC, Kainberger F, Weber M, Nemec S, et al. Effects of unloading and compression on T1-Gd |(dGEMRIC) relaxation times in healthy articular knee cartilage: in vivo effects at three tesla [Abstract]. Skeletal Radiol. 2009;38:631.

6. Willi JP, Merlini L, Combescure C, Rager O, Crook DW, Imperiano $\mathrm{H}$, et al. Comparison of diffusion weighted and dynamic enhanced subtracted MRI with bone scintigraphy in the diagnosis and prognosis of Perthes disease [Abstract]. Skeletal Radiol. 2009;38:615.

7. Kotnis NNA, Davies AM, James SSLJ. Normal post-operative MRI appearances following hindquarter amputation [Abstract]. Skeletal Radiol. 2009;38:615-6.

8. Kotnis NNA, Davies AM, James SSLJ. The MRI appearances of local disease recurrence following hindquarter amputation [Abstract]. Skeletal Radiol. 2009;38:609.

9. Horak M, Ryznarova Z, Kaspar M. CT perfusion can effectively monitor the effect of chemotherapy in patients with osteosarcoma [Abstract]. Skeletal Radiol. 2009;38:622.

10. Wick MC, Weiss RJ, Jaschke W, Klauser AS. Erosions, but not bone marrow oedema or contrast medium enhancement, are specific features in MRI of ankylosing spondylitis patients [Abstract]. Skeletal Radiol. 2009;38:603.

11. Huetink K, Nelissen RGH, Van Erkel AR, Watt I, Bloem JL. MRI on radiographic findings after 10 years in patients with non-acute knee complaints; determination of risk for the development of osteoarthritis [Abstract]. Skeletal Radiol. 2009;38:619.

12. Madan-Sharma R, Watt I, Oostveen AJCMA, Viergever M, Bloem JL, Kloppenburg M. Magnetic resonance imaging characteristics of knees of a healthy population and of early knee osteoarthritis and the relation to pain and disability [Abstract]. Skeletal Radiol. 2009;38:614-5.

13. Van Rillaer O, Morales L, Westhovens R, Neven P, Pans S. MRI imaging findings in early-stage breast cancer patients treated with aromatase inhibitors developing AI-associated arthralgia syndrome [Abstract]. Skeletal Radiol. 2009;38:626-7.

14. De Conti G, Angelini F, Marchioro U, Boscolo N, Trevisan M, Vio S. Clinical and MRI follow-up in 123 consecutive patients after percutaneous ultrasound guided ablation of shoulder tendons calcifications [Abstract]. Skeletal Radiol. 2009;38:613.

15. De Zordo T, Ahmad N, Girtler MT, Klauser AS, Chhem R, Romagnoli C. Outcome of ultrasound-guided aspiration and lavage in calcific tendinosis of the shoulder and other tendons [Abstract]. Skeletal Radiol. 2009;38:625-6.

16. Flood K, Hopper M, O'Connor P, Barron D, Rankine J, Robinson $\mathrm{P}$, et al. Plantar fascia injections: does technique matter? [Abstract] Skeletal Radiol. 2009;38:628-9.

17. Sinha A, Mctiernan R, Wilson DJ. Ultrasound guided aspiration for painful hips in children; should it be performed? [Abstract] Skeletal Radiol. 2009;38:614. 